\title{
Los libros de coro copiados por fray Miguel de Aguilar: un primer acercamiento al estudio de su encuadernación en la Nueva España
}

\author{
Tania Estrada Valadez \\ Patricia de la Garza Cabrera \\ Thalía Edith Velasco Castelán
}

a estabilización, el registro y el estudio del acervo de libros de coro del Museo Nacional del Virreinato (MNV), perteneciente al Instituto Nacional de Antropología e Historia (INAH), México, nos permitió identificar un conjunto de volúmenes escritos por un mismo calígrafo novohispano, ${ }^{1}$ hallazgo que nos impulsó a analizar las características de los libros elaborados en la Nueva España entre los años 1700 y 1719 por el copista fray Miguel de Agui$\operatorname{lar}^{2}$ y, con ello, conocer su técnica de manufactura y materiales constitutivos.

\section{Antecedentes}

El libro de coro de 1715 del Museo Nacional de Historia, MNH-INAH, (LC1715-MNH) ${ }^{3}$ Ilegó en 1973, para su restauración, a la Coordinación Nacional de Conservación del Patrimonio Cultural (CNCPC), entonces Departamento de Conservación del Patrimonio Cultural, según datos registrados en el oficio de entrada (CNCPC-INAH 1973). No fue sino hasta el 2011 cuando la investigación del libro se retomó como tema de tesis (Estrada y De la Garza 2013); durante

\footnotetext{
${ }^{1}$ El proyecto "Estabilización de la colección de los libros de coro del Museo del Virreinato [MNV], Tepotzotlán", ejecutado por la Coordinación Nacional de Conservación del Patrimonio Cultural (cncpc-inah) en el 2013, en colaboración con el MNV y el Centro Nacional de Investigación, Documentación e Información Musical (Cenidim-Conaculta), tuvo el objetivo de estabilizar y digitalizar este importante acervo.

${ }^{2}$ La identificación de estos libros, a los que se suman cuadernillos, se realizó no sólo gracias a la presencia del colofón, sino también a la referencia, con algunas letras iniciales, del copista dentro del contenido de los libros. Colofón: fórmula final de un códice en la que el copista mencionaba el lugar, la fecha de la copia o ambos (Martínez de Sousa 2004:198).

${ }^{3}$ En adelante, a los libros se les asignará una clave que hace referencia a la siguiente información: las siglas del objeto (libro de coro, LC); posteriormente, el año de su manufactura y, finalmente, el acervo en el cual se resguarda. Esta referencia se retoma de la tesis de Estrada y De la Garza (2013).
} 
el desarrollo, en conjunto con el de otras investigaciones y proyectos que se han generado en torno de los libros corales en nuestro país, ${ }^{4}$ identificamos un importante conjunto de volúmenes escritos por el mismo calígrafo: fray Miguel de Aguilar. Los quince libros de coro elaborados por este copista hoy están resguardados en distintas colecciones: uno pertenece al MNH-INAH, dos a la Biblioteca Nacional de México de la Universidad Nacional Autónoma de México (BN-UNAM) y doce al MNV. ${ }^{5}$

\section{Estado de la cuestión 6}

Para comprender el contexto histórico, uso y función de los libros de coro se hizo una revisión bibliográfica de los estudios e investigaciones sobre este tipo de bienes documentales. En nuestro país, por un lado, este tipo de libros se ha estudiado desde el punto de vista del contenido y su función social (Romero de Terreros 1943a; Tello 1988; Lara Cárdenas 1996; Marín 2007; Velasco Castelán 2009a y Salgado Ruelas 2010), sin atender a detalle la técnica de manufactura y los materiales constitutivos $y$, por el otro, son escasas las investigaciones sobre el oficio e historia de la encuadernación (Estrada y De la Garza 2013:5).

Peor aún, tras la revisión de las fuentes bibliográficas (Bueno 2005; Velasco Castelán 2009b; Pérez 2009; García Isac, Lidón y Sánchez 2010) dedicadas a los libros de coro mexicanos, determinamos que existen vacíos en dos sentidos. El primero es la casi nula información e interpretación de los datos obtenidos sobre la estructura y materialidad de las encuadernaciones y el segundo, la poca vinculación de tales datos con las propuestas de intervención de estos bienes. Cabe señalar que el estudio arqueo-

\footnotetext{
${ }^{4}$ La identificación de los libros de coro recoge el importante trabajo realizado por investigadores y proyectos de distintas instituciones. Ejemplos de ello fueron el proyecto de "Estabilización y digitalización de los libros de coro de la Catedral de México", y un inventario de los libros del MNV, actividades enmarcadas en el Seminario Nacional de Música en la Nueva España y México Independiente, del Instituto de Investigaciones Estéticas de la Universidad Nacional Autónoma de México (IIES-UNAM). La tesis doctoral de Silvia Salgado Ruelas sobre los libros de coro de la bn también identificó dos libros de este calígrafo (Salgado Ruelas 2010). Asimismo, en el 2007 la restauradora Thalía E. Velasco Castelán elaboró un dictamen de la colección de los libros de coro del MNV, en el cual identificó la existencia de varios ejemplares elaborados por fray Miguel de Aguilar (Velasco Castelán 2005:37), y posteriormente analizó y publicó un artículo en el que abordó la técnica de manufactura del libro que se encontraba en la cncpc (Velasco Castelán 2009b:347-364).

${ }^{5}$ Actualmente hemos identificado sólo doce libros de coro en el MNV, aunque probablemente fray Miguel de Aguilar elaboró un mayor número de volúmenes, pues en este museo se conservan dos cuadernillos sueltos con su firma que nos permiten suponer que produjo, al menos, diecisiete libros.

${ }^{6}$ Este apartado se realizó con base en la revisión bibliográfica de la introducción de la tesis de Estrada y De la Garza (2013:5-6).
}

lógico $^{7}$ del libro, es decir, como artefacto, es reciente en México, por lo que ha de considerarse que nuestro trabajo ha aportado información novedosa sobre la encuadernación de los libros de coro del siglo XVIII en la Nueva España.

\section{Construcción, uso y traslados durante tres siglos}

Como parte del trabajo de investigación, se realizaron indagaciones documentales para rastrear los orígenes y procedencia de los libros estudiados. La presencia del colofón, ${ }^{8}$ con la fecha de manufactura y los datos del comitente $^{9}$ y del copista, o scriptor, ${ }^{10}$ es un elemento del libro que aporta información relativa a su manufactura.

Como ya se mencionó, se han localizado quince libros de coro copiados por fray Miguel de Aguilar, incluidos dos cuadernillos, que contienen un colofón, ${ }^{11}$ gracias a lo cual se logró identificar al personaje y los volúmenes que produjo, amén de la referencia del copista en algunas letras iniciales dentro del contenido de las obras. ${ }^{12}$ Esta información se vio enormemente enriquecida con el hallazgo de un oficio emitido por Antonio Cortés en 1915 (Cortés 1915), trabajador del Museo Nacional de Arqueología, Historia y Etnología (MNAHE), México, quien hizo un inventario de los objetos que la BN donaría a este museo (Estrada y De la Garza 2013:26).

Si bien la mayoría de los colofones analizados tienen un mismo estilo caligráfico y de contenido, en cuanto a su formato, varían la forma y el estilo del marco que los rodea: en casi todos los casos es rectangular o cuadrada, definida con un diseño en líneas quebradas, pero en ocasiones presenta formas como la circular o en rombo, con

\footnotetext{
${ }^{7}$ Ligatus define la arqueología del libro como "registro consistente y ordenado de las características individuales, observadas en cada objeto con el fin de facilitar la descripción de la encuadernación y la interpretación de los datos obtenidos". Ligatus es un grupo de investigadores que tienen como objeto estudiar la encuadernación de los libros desde un punto de vista histórico y de conservación con ayuda de herramientas digitales (Ligatus 2011).

${ }^{8}$ Fórmula final de un códice en la que el copista mencionaba el lugar, la fecha de la copia o ambos (Martínez de Sousa 2004:198).

${ }^{9}$ Persona que encargaba y financiaba el trabajo (Estrada y De la Garza 2013:22).

${ }^{10}$ Scriptor, término en latín, también Ilamado amanuense: aquella persona que tiene por oficio escribir a mano, copiando o poniendo en limpio escritos ajenos o escribiendo lo que se le dicta (RAE 2013).

${ }^{11}$ El estudio que aquí se realiza se aboca a los libros de coro; por el momento no estudiamos la manufactura de los cuadernillos.

${ }^{12}$ La mayoría de los libros de coro no contiene datos relativos a su manufactura, de allí que la existencia de un colofón o la referencia del scriptor en las letras iniciales resulten un gran aporte, pues revela información sobre su procedencia. Si bien este punto debe estudiarse con mayor detenimiento, el acercamiento a la colección de los libros de coro de la Catedral de México y del MNV nos permite afirmar que más de $60 \%$ de los libros de estos acervos carece de referencias de su fecha de manufactura y del copista.
} 
líneas en tinta; respecto de su contenido, prácticamente en todos es constante: además de mencionar el nombre del copista, fray Miguel de Aguilar, se hace referencia al comitente y al año en el que se elaboró.

De acuerdo con los datos bibliográficos recabados, se sabe que el copista fray Miguel de Aguilar era "español", originalmente (a fines del siglo XVII) miembro de la provincia $^{13}$ de Michoacán; ${ }^{14}$ en 1701 fue trasladado a la provincia de México, donde permaneció hasta 1720, año en el que fue reubicado en Lolotla, Hidalgo (Ruiz 1984:140 y 407). El periodo comprendido entre estos dos años, en el que podemos asegurar que De Aguilar se encontraba en la ciudad de México (Estrada y De la Garza 2013:20-28), coincide con la mayoría de las fechas de producción de los quince libros mencionados.

Así, podemos sugerir, por una parte, que el libro más antiguo elaborado por De Aguilar que hoy conservamos, escrito en 1700, es el único que no se produjo en la ciudad de México, mientras que el resto, fabricados en $1701,1706,1707,1708$, dos en 1713 , tres en 1714 , $1715,1716,1718$ y dos en $1719,{ }^{15}$ probablemente sí.

De acuerdo con la información citada por fray Alipio Ruiz, entre 1714 y 1717 fray Miguel de Aguilar tuvo también el cargo de definidor, es decir, formaba parte del cuerpo consultivo de la gestión de las provincias, ${ }^{16}$ dato del que se infiere que algunos copistas no se dedicaban únicamente al trabajo artesanal de copiar los libros, lo que significa que De Aguilar, además de elaborar seis en este periodo - tres en 1714, uno en 1715 y dos en 1716-, realizaba actividades administrativas dentro del convento (Ruiz 1984:140 y 407).

Como ya se dijo, otro dato registrado en los colofones es el nombre del comitente, así como su cargo dentro de la comunidad: si era provincial ${ }^{17}$ o definidor. El primer comitente identificado en los libros de coro fue fray Gaspar Ramos, "español", provincial de México entre 1699 y 1702 (Ruiz 1984:140 y 407): coinciden con estas fe-

\footnotetext{
13 "La provincia era una entidad jurídica formada por varios conventos (Ilamados prioratos entre los dominicos y los agustinos) distribuidos sobre un territorio y sujetos al gobierno de un provincial (Rubial 2005:189-190).

${ }^{14}$ Las dos provincias de agustinos: la del santísimo nombre de Jesús, en México, y la de San Nicolás Tolentino, en Michoacán (Rubial 1989:86107).

${ }^{15}$ En esta relación no se tomaron en cuenta los cuadernillos que también se conservan, uno de 1711 y otro de 1716 .

${ }^{16}$ Los agustinos tenían una autogestión, es decir, elegían a sus cuerpos rectores. El provincial, que regía los destinos de la comunidad durante tres años y los cuatro definidores que le ayudaban en el gobierno, eran electos por los priores y discretos de todas las casas de la provincia reunidos en capítulo (Rubial 1989:42).

17 "El provincial tenía en sus manos la organización de la misión, labor que compartía con el definitorio al cual debía pedir consejo y aprobación aunque a veces se arrojaba funciones extras" (Rubial 1989:44).
}

chas tanto el LC-1700-MNV ${ }^{18}$ como el LC-1701-MNV. La mayoría de aquéllos —siete ejemplares: LC-1707-MNV, C-1711-MNV, ${ }^{19}$ LC-1713-MNV, LC-1713-BN, LC-1714BN, LC-1714-MNV y LC-1715-MNH- fueron encargados por fray Antonio Gutiérrez, "español", natural de Medina del Campo (provincia de Castilla, España), miembro del convento de Salamanca, España, quien fuera presidente de capítulo de la provincia de México a la fecha de su fallecimiento, el 3 de marzo de 1715 (Ruiz 1984:140). El tercer comitente registrado es Baltasar Sánchez, nacido también en la Península Ibérica en 1669, quien solicitó el cuadernillo C-1716-MNV y los libros LC-1716-MNV, LC1718-MNV, LC-1719-MNV1 y LC-1719MNV2, según sus respectivos colofones. ${ }^{20}$

Manuel Romero de Terreros afirma en La iglesia y convento de san Agustín (Romero de Terreros 1943b) que fray Miguel de Aguilar — cuya labor como copista destacaprodujo la mayoría de estos libros en el convento de san Agustín en la ciudad de México entre 1701 y 1719, donde, puntualiza, los propios frailes los escribían. Otro elemento que confirma esta procedencia es la existencia, en un gran número de volúmenes de este conjunto, de un chapetón ${ }^{21}$ central con la representación del símbolo agustino $^{22}$ (corazón atravesado por dos flechas en cruz), alrededor del cual se observan otros cuatro chapetones redondos, cuya figura principal es una flor, que forman un rombo; a su vez, presentan esquineras, asimismo con el símbolo agustino, protecciones de cantos al pie y a la cabeza, y dos broches en el canto de frente de las tapas (Figura 1).

Durante más de siglo y medio, ${ }^{23}$ los libros de coro servían al interior del convento para la celebración del oficio divino, ${ }^{24}$ actividad que se deduce de las huellas de uso que observamos en los cuerpos de los libros, tales como manchas y roturas en las esquinas de canto de frente, grietas y suciedad en las esquinas inferiores de los

\footnotetext{
${ }^{18}$ El objeto de la clasificación de los volúmenes es identificar claramente cada uno de ellos, en lugar de utilizar su número de inventario INAH. ${ }^{19}$ En adelante se identificarán los cuadernillos con la letra C, posteriormente, como en el caso de los libros, se referirá el año de su manufactura y finalmente se anota el acervo en el cual se resguarda.

${ }^{20}$ Sánchez fue definidor suplente entre 1705 y 1708, definidor de 1708 a 1711 y, finalmente, provincial, de 1711 a 1714 (Ruiz 1984:407). De acuerdo con esta información, se puede sustentar que el comitente no tenía que ser necesariamente alguien con un cargo administrativo.

${ }^{21}$ Chapetón (también Ilamado clavazón o guarnición): cualquier pieza de metal que guarnece la encuadernación (Estrada y De la Garza 2013:116).

${ }^{22}$ Atributo de san Agustín que expresa contrición (Ferguson 1956:55).

${ }^{23}$ A diferencia de los libros corales de la Catedral de México, que se utilizaron aún hasta casi el final del siglo XIX, los de los conventos fueron recogidos en la segunda mitad de ese siglo por el Estado, a raíz de las leyes de supresión y desamortización de los bienes eclesiásticos (Salgado Ruelas 2010:20).

${ }^{24}$ Aunque, como se verá en las reflexiones finales, pudieron tener otros usos.
} 


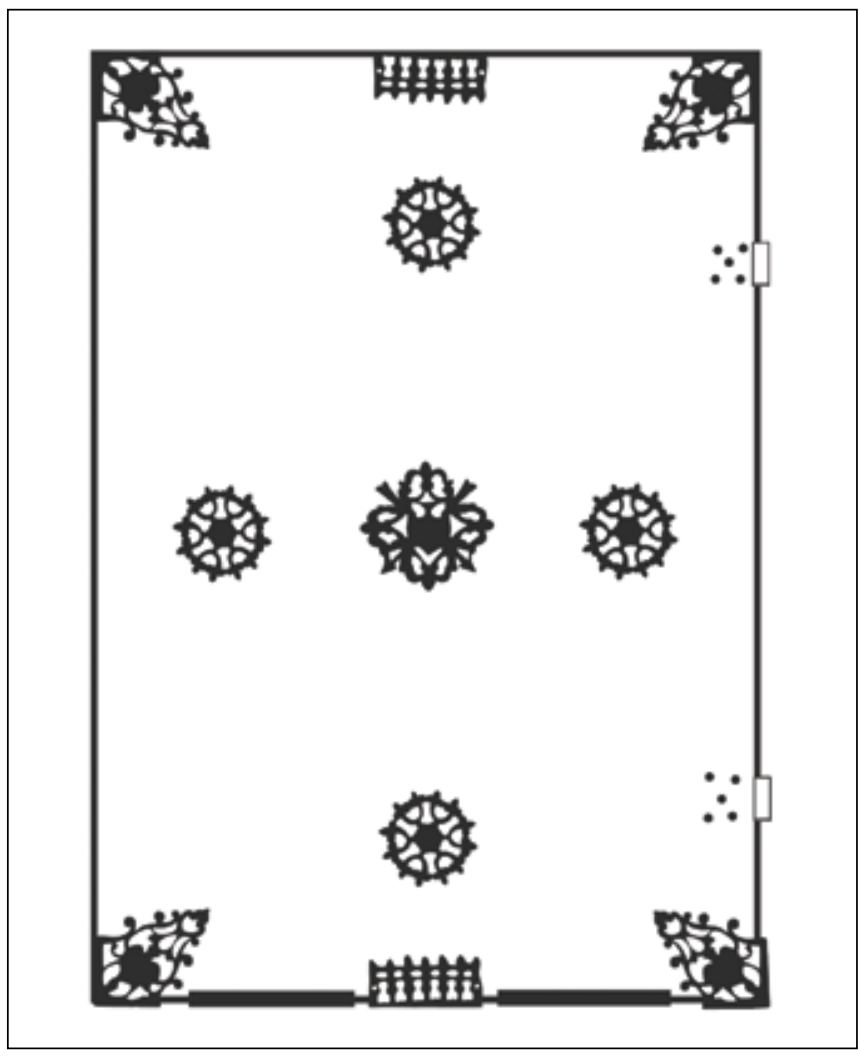

FIGURA 1. Tapa con chapetones (Esquema: Patricia de la Garza 2013; fuente: Estrada y De la Garza 2013:88).

folios, efecto del constante movimiento de las hojas y goteo de cera por el uso de velas. También son evidentes reparaciones históricas, como parches, costuras, raspado de pergamino y repintes, así como injertos en el material de recubrimiento, elaboración de nuevas costuras y reencuadernaciones completas.

A partir de 1859, año en que se decretó la ley de nacionalización de los bienes del clero, numerosos acervos documentales y bibliográficos conventuales pasaron a propiedad del gobierno mexicano, entre ellos, los de San Agustín (Ruiz Castañeda et al. 2004:481). En 1867 se decretó la conversión del antiguo convento de San Agustín en sede de la nueva Biblioteca Nacional, lo que explica que la colección de libros de coro de este recinto religioso se conservara unida (Salgado Ruelas 2010:19).

En la década de 1910 se le solicitó a la BN la donación de distintos objetos al MNAHE, entre los que se encontraba un conjunto de libros de coro. Una vez realizadas algunas gestiones, en 1915 se encomendó a Antonio Cortés, empleado del MNAHE, que realizara una visita a la BN para ubicar y enumerar los objetos que se trasladarían al museo para su exhibición.

El oficio de Antonio Cortés que se conserva en la $\mathrm{BN}^{25}$ describe nueve libros de coro que, se asegura, realizó

${ }^{25}$ Gracias a la localización del documento en la bn se logró explicar su tránsito de ésta al MNV (Velasco Castelán 2012:157-159). fray Miguel de Aguilar -es decir, no los quince que hoy se tienen del copista (Cortés 1915)—, de los cuales se han localizado ocho. El único que hace falta localizar tiene como referencia la fecha de manufactura (1710), así como la siguiente información: "En 2 de marzo de 1710 (se empezó el 2 de marzo y se acabó el 29 del mismo mes)" (Figura 2).

Los datos que se incluyen en este documento son muy generales: en ocasiones sólo contamos con el año de producción del libro, lo que dificulta relacionarlo con cada ejemplar, mientras que los casos en los que se hacen referencias de algún dato complementario (como, por ejemplo, "tiene la fecha en la página 23": LC-1701-MNV) o de algún deterioro característico ("sin la portada ni las ocho primeras páginas": LC-1713-MNV) nos permitieron identificar el volumen con mayor facilidad (Figura 2).

Si bien Antonio Cortés emitió dicho oficio en 1915 (Cortés 1915), no se sabe si efectivamente en ese año se efectuó el traslado de los libros, pues no se ha localizado ningún documento en el que estén asentadas la salida de las piezas de la BN ni su entrada al MNAHE. No se tiene noticia, sino hasta 1925, del ingreso de aproximadamente treinta y siete libros de coro al MNAHE, descrito en un oficio del Archivo Histórico del Museo Nacional de Antropología (AHMNA), aunque no se tiene la lista específica de los libros corales (AHMNA-INAH 1925: f.124). Del conjunto de los copiados por de Aguilar únicamente dos, el LC-1713-BN y el LC-1714-BN, se conservaron en la BN.

Gracias al descubrimiento de estos dos documentos podemos afirmar que los libros de coro estaban resguardados en la BN y que probablemente en la década de 1920 se trasladaron al MNAHE, en el que permanecieron durante varias décadas para, posteriormente, incorporarse a los acervos del MNV y el MNH.

En el caso específico del volumen LC-1715-MNH, sabemos que fue desplazado al $\mathrm{MNH}$, fundado en 1954 (Olivé Negrete y Cottom 2003:34), en el que se conservó hasta octubre de 1973, fecha en la que fue llevado al entonces Departamento de Restauración del Patrimonio Cultural (CNCPC 1973).

En 1964 se creó el MNV, recinto al que se trasladaron los libros LC-1700, LC-1701, LC-1706, LC-1707, LC1708, C-1711, LC-1713, LC1-1714, LC2-1714, C-1716, LC-1716, LC-1718, LC1-1719 y LC2-1719²6 (Lara Cárdenas 1996:52).

\section{Análisis de los libros de coro}

El obtener información relativa a la manufactura de la encuadernación de estos libros de coro fue uno de los motivos que nos impulsó a realizar un registro exhaustivo de materiales, técnicas decorativas, características y elemen-

\footnotetext{
${ }^{26}$ El MNV resguarda 92 libros de coro, acervo que se conformó al reunir volúmenes de distinta procedencia: MNA, Catedral de México y otros recintos religiosos (Lara Cárdenas 1996:52).
} 


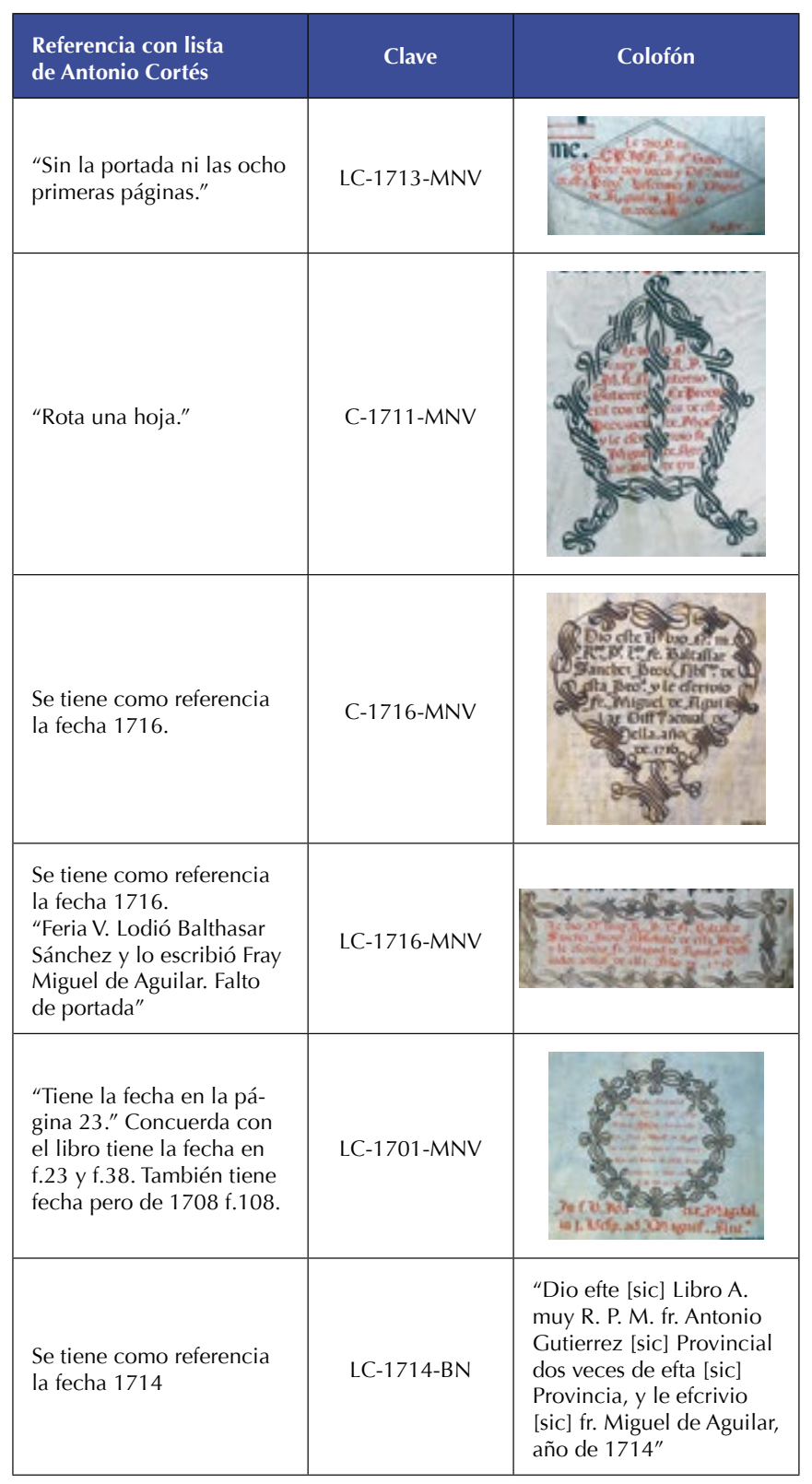

\begin{tabular}{l|l|l|}
\hline $\begin{array}{l}\text { Referencia con lista } \\
\text { de Antonio Cortés }\end{array}$ & Clave \\
\hline $\begin{array}{l}\text { Se tiene como referencia } \\
\text { la fecha 1714. }\end{array}$ & LC-1714-MNV1 \\
\hline $\begin{array}{l}\text { Se tiene como referencia } \\
\text { la fecha 1714. }\end{array}$ & LC-1714-MNV2 \\
\hline $\begin{array}{l}\text { "En 2 de Marzo de 1710 } \\
\text { "se empezó el 2 de marzo }\end{array}$ & LC-1710 \\
y se acabó el 29 del \\
mismo mes."
\end{tabular}

FIGURA 2 Libros de coro localizados en la lista de Antonio Cortés (Tabla: Estrada 2013; fuente: Estrada, De la Garza y Velasco 2014 ; digitalización original: Ricardo Castro (CNCPC-INAH); diseño del gráfico: Jorge Alejandro Bautista Ramírez).

tos estructurales, así como a elaborar su análisis comparativo. Aunque podíamos afirmar que todos los volúmenes aquí identificados habían sido copiados por fray Miguel de Aguilar, carecíamos de información respecto de su encuadernación, y fue así como surgieron las siguientes interrogantes: ¿estas encuadernaciones las ejecutaron distintos artesanos? El encuadernador o el taller de encuadernación ¿era de la Orden de los agustinos o se trataba de laicos que trabajaban en un taller ajeno a ella?

Es importante aclarar que, no obstante el estudio derivado de tales cuestionamientos se enfoca en el análisis de la encuadernación, el dato por el que identificamos y buscamos relaciones entre el conjunto fue el del nombre del copista, a partir del cual deducimos que lo más probable es que únicamente copiara los libros, ${ }^{27}$ esto es - sin poder asegurarlo-, que no estuvo involucrado en el proceso de elaboración de la encuadernación, que probablemente se llevó a cabo en el convento de San Agustín, lugar donde laboraba y, según las evidencias, se elaboraron los libros. De ahí que el estudio de estas encuadernaciones sea de gran valor, pues nos permitirá

\footnotetext{
${ }^{27}$ El análisis del estilo, materiales, técnica, características y estado de conservación de los elementos caligráficos y las iluminaciones de este conjunto de libros novohispanos de principios del siglo XVIII es una línea de investigación que queda pendiente, en tanto requiere un acercamiento interdisciplinar y escapa a los alcances del trabajo que aquí se presenta.
} 
comparar y conocer las características de un grupo de volúmenes producidos en la Nueva España en las primeras dos décadas del siglo XVIII.

Una vez identificados los libros referidos, se realizó el mencionado análisis comparativo de las características estilísticas y materiales de la encuadernación, para lo cual se retomó tanto la ficha de descripción propuesta en la tesis de Estrada y De la Garza (2013:211-232) como la ficha del proyecto "Estabilización de la colección de los libros de coro del Museo Nacional del Virreinato, Tepotzotlán". Cada ejemplar se clasificó en cinco grupos, que se presentan en la siguiente tabla (Figura 3).

Por ahora, si bien nos hemos enfocado en aquellos libros que presentan la primera encuadernación -probablemente elaborada en una época cercana a la de su escritura-, pues son lo que nos pueden otorgar más información sobre sus materiales y estructura, cabría afirmar que la totalidad de los volúmenes nos podrían aportar conocimiento sobre la manufactura de los libros (Figura 4).

\section{Descripción formal y técnica de factura}

La información que a continuación se presenta es sólo el primer acercamiento en cuanto a manufactura de la en- cuadernación del conjunto de cuatro libros que presentan la primera encuadernación:

\section{a) Cuerpo del libro}

- Este conjunto de libros se distingue por su gran formato: son los más grandes que existen en la colección de los libros de coro del MNV. El largo de las cuatro encuadernaciones supera los $900 \mathrm{~mm}$ y el ancho, los $650 \mathrm{~mm}$. Otro de los elementos compositivos que caracteriza el trabajo de este copista es la presencia de márgenes amplios, que rondan los 176 X 189 mm.

- El cuerpo del libro está integrado por cuadernillos de pergamino, en los que siempre se mantiene la regla de Gregory $^{28}$ (Figura 5). Por lo anterior, se deduce que, antes de que el copista transcribiera el texto, tenía planeados tanto su orden y distribución como la cantidad de hojas de pergamino que se requerían.

\footnotetext{
${ }^{28}$ Según la regla de Gregory, las páginas en pergamino contiguas deben tener la misma coloración cuando el libro esté abierto, es decir, las mismas caras del soporte (flor o carnaza) han de coincidir en posición enfrontada a lo largo de todo el cuerpo (vuelto y recto) (Ruiz García 2002:147-148).
}

\begin{tabular}{|c|c|c|c|c|c|c|}
\hline \multicolumn{7}{|c|}{ Clasificación de los libros de coro de Fray Miguel de Aguilar } \\
\hline \multirow[b]{2}{*}{1} & \multicolumn{6}{|c|}{ Conservan su primera encuadernación } \\
\hline & $\begin{array}{c}\text { LC-1714- } \\
\text { MNV1 }\end{array}$ & LC-1715-MNH & $\begin{array}{c}\text { LC-1719- } \\
\text { MNV1 }\end{array}$ & $\begin{array}{l}\text { LC-1719- } \\
\text { MNV2 }\end{array}$ & & \\
\hline \multirow{2}{*}{2} & \multicolumn{6}{|c|}{ Conservan su primera encuadernación con reparaciones } \\
\hline & LC-1701-MNV & LC-1708-MNV & LC-1713-MNV & LC-1714-BN & LC-1718-MNV & \\
\hline \multirow{2}{*}{3} & \multicolumn{6}{|c|}{ Fueron reencuadernados entre el siglo XVIII y XIX } \\
\hline & \multicolumn{6}{|l|}{ LC-1716-MNV } \\
\hline \multirow{2}{*}{4} & \multicolumn{6}{|c|}{ Fueron reencuadernados en una época cercana a la actual ("contemporánea") } \\
\hline & LC-1713-BN & & & & & \\
\hline \multirow{3}{*}{5} & \multicolumn{6}{|c|}{ Perdieron su encuadernación } \\
\hline & \multirow{2}{*}{ LC-1700-MNV } & \multirow{2}{*}{ LC-1706-MNV } & \multirow{2}{*}{ LC-1707-MNV } & \multirow{2}{*}{$\begin{array}{c}\text { LC-1714- } \\
\text { MNV2 }\end{array}$} & \multicolumn{2}{|c|}{ Cuadernillos sueltos } \\
\hline & & & & & C-1711-MNV & C-1716-MNV \\
\hline
\end{tabular}

FIGURA 3. Los libros de coro de fray Miguel de Aguilar clasificados en cinco categorías (Tabla: Estrada, De la Garza y Velasco Castelán 2014). 


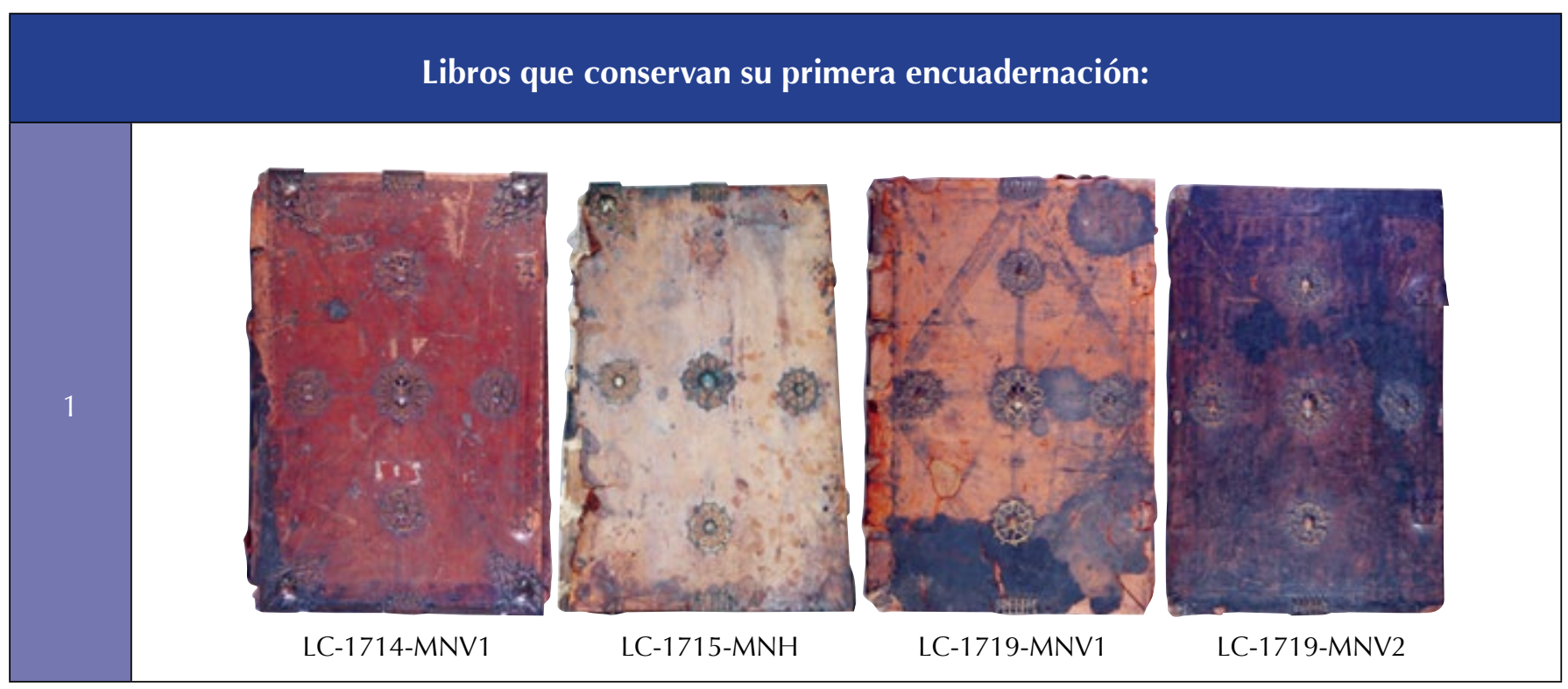

FIGURA 4. Libros que conservan su primera encuadernación (Fotografía: Ricardo Castro 2013; cortesía: INAH).

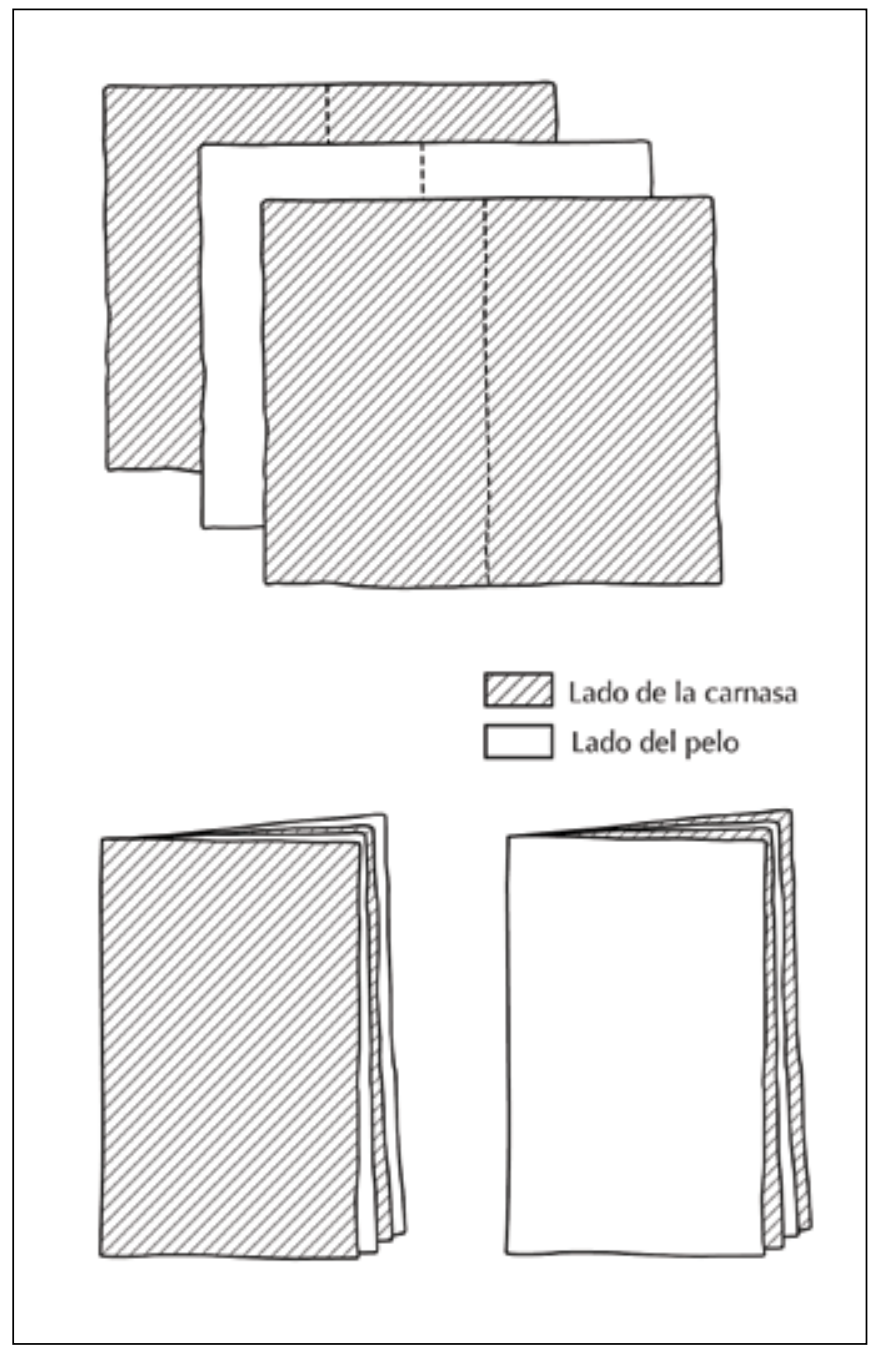

FIGURA 5. Regla de Gregory (Esquema: Patricia de la Garza 2013; fuente: Estrada y De la Garza 2013:36).
- La construcción de las fojas es similar en todos los casos: formadas al adherir dos hojas por medio de escartivanas, o talones, ${ }^{29}$ con la prolongación del mismo pergamino (Figura 6). Casi todos los cuadernillos de pergamino están compuestos por tres fojas (seis hojas en total).

- Las guardas se encontraban cosidas al primer y último cuadernillos; sin embargo, en la mayoría de los casos parecen haber sido sustraídas del volumen, excepto en el LC-1715-MNV, que conserva la guarda posterior de pergamino de reúso. ${ }^{30}$ Llama la atención que tres de los cuatro volúmenes no tienen las guardas (anterior y posterior), aunque se conserva el talón; probablemente deben haber sido reutilizadas, debido a su costo o a su escasez.

- Los cantos de los libros son lisos, regulares y sin decoración.

\section{b) Encuadernación}

b.1) Estructura:

- La costura de los cuatro libros analizados es seguida, no compensada, con siete estaciones de costura: ca-

\footnotetext{
${ }^{29}$ Talón: "Se refiere a una pestaña que se pliega a lo largo de la hoja a manera de escartivana con la finalidad de permitir la adhesión a otra hoja con una pestaña similar para formar una foja" (Estrada y De la Garza 2013:117).

${ }^{30}$ El reúso de las hojas de pergamino y papel era una práctica común para ciertos elementos de los libros, como las guardas, las contraguardas, el endose y las almas de las cabezadas (Hellinga 2000; Pedraza Gracia et al. 2003; Romero en prensa).
} 


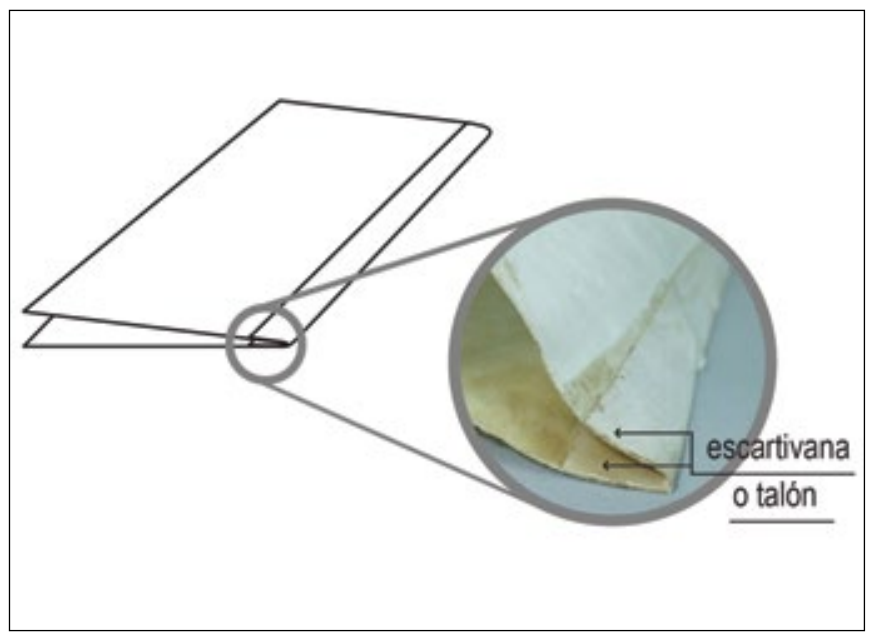

FIGURA 6. Escartivana o talón (Esquema: Patricia de la Garza 2013; fuente: Estrada y De la Garza 2013:33).

denetas al pie y a la cabeza, y cinco nervios dobles de fibras vegetales similares a una cuerda, distribuidos a intervalos regulares a lo largo de la lomera (Figura 7).

- Los cuatro libros tienen lomera en media caña y cabezadas tejidas con costura compensada. Las almas son de fibra y sus prolongaciones, que están trenzadas, también se enlazan a las tapas.

- En todos los casos el endose es fraccionado, transversal y de nervio a nervio; los bordes de sus prolongaciones están adheridos a las tapas.

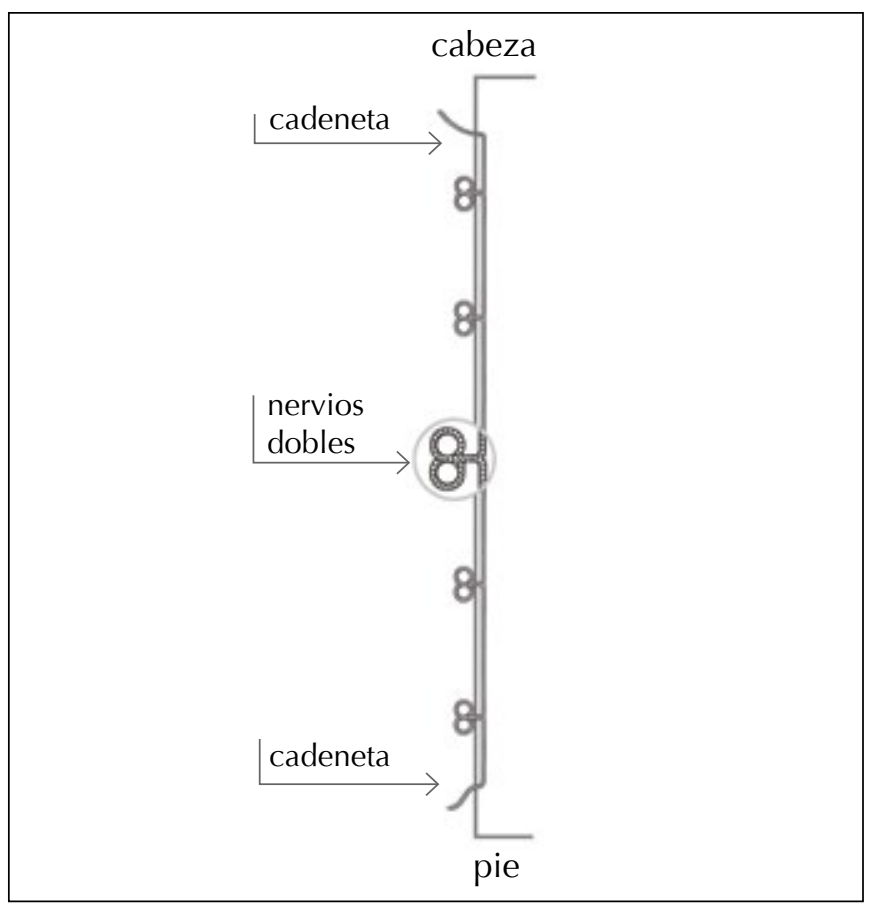

FIGURA 7. Tipo de costura (Esquema: Patricia de la Garza 2013; fuente: Estrada y De la Garza 2013:51).
- Ambas tapas presentan siete alojamientos en el exterior y en el interior, los cuales tienen la función de albergar las prolongaciones de los nervios y de las almas de las cabezadas (Figura 8). Se puede inferir que cada tapa está formada por piezas unidas por medio de ensambles de caja y espiga, y reforzadas, posiblemente, con adhesivo de origen animal.

b.2) Material de recubrimiento y elementos metálicos:

- Los libros presentan, salvo uno, una encuadernación entera de piel lisa de color café.

- Las encuadernaciones cuentan con chapetones, esquineras y elementos metálicos en los cantos de las tapas. Los cuatro chapetones se encuentran dispuestos alrededor de un chapetón central con el símbolo agustino semejando un rombo. En todos los libros había ocho esquineras, cuatro ubicadas en cada esquina de ambas tapas, labradas con motivos de la Orden agustina (Figura 9).

- En cada uno de los volúmenes únicamente se conservan algunos fragmentos de los broches, que estaban ubicados en el canto de frente de la tapa anterior sobre el canto de frente.

- Con excepción del LC-1715-MNH, los demás libros presentan gofrado ${ }^{31}$ en los planos de las tapas, mientras

${ }^{31}$ Cofrado: “Decorado de una superficie con estampado en seco, en hueco o en relieve, mediante hierros calientes" (Estrada y De la Garza 2013:118).

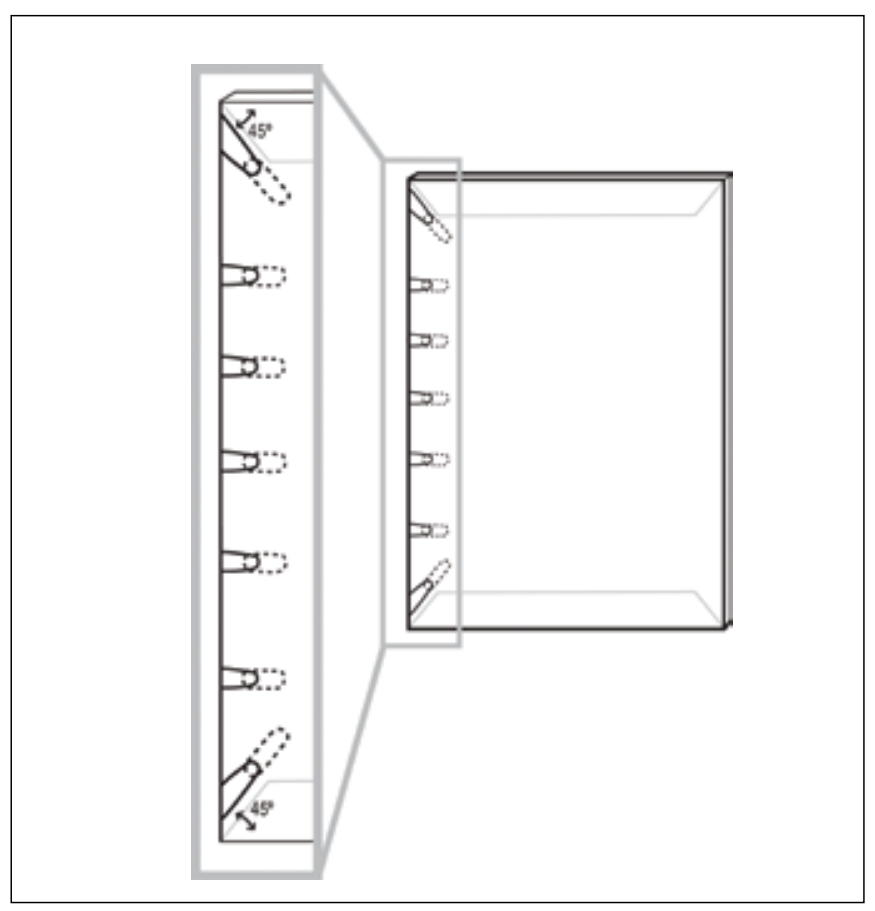

FIGURA 8. Alojamientos (Esquema: Patricia de la Garza 2013; fuente: Estrada y De la Garza 2013:75). 


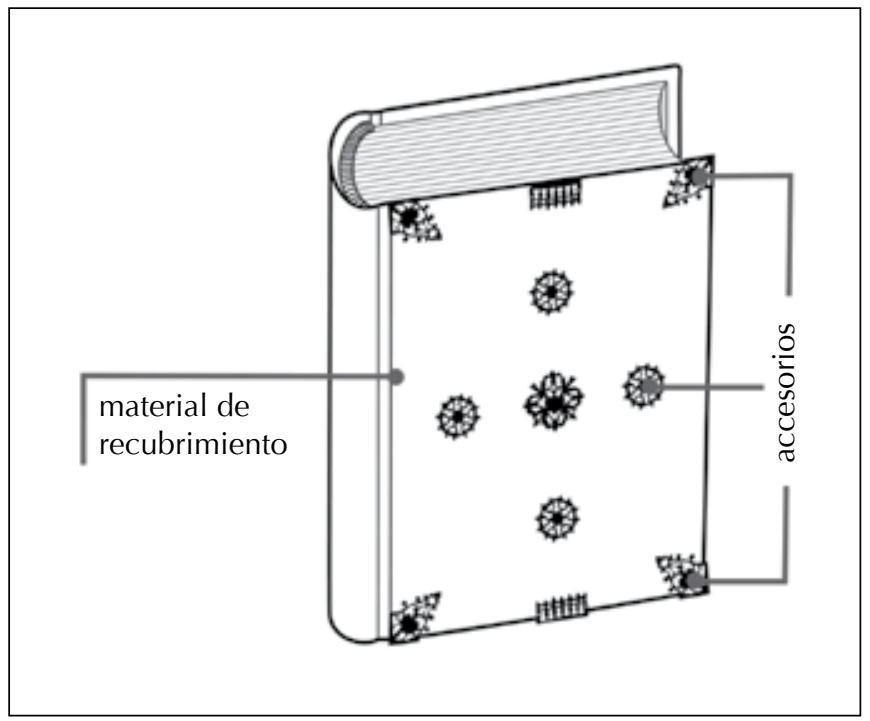

FIGURA 9. Material de recubrimiento y accesorios (Esquema: Patricia de la Garza 2013; fuente: Estrada y De la Garza 2013:30).

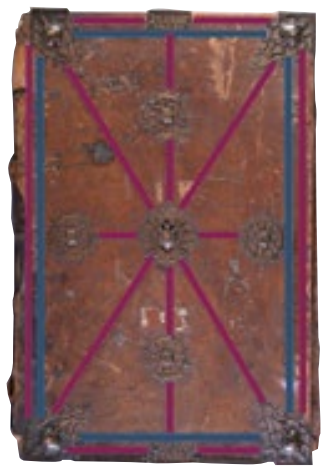

LC-1714-MNV1

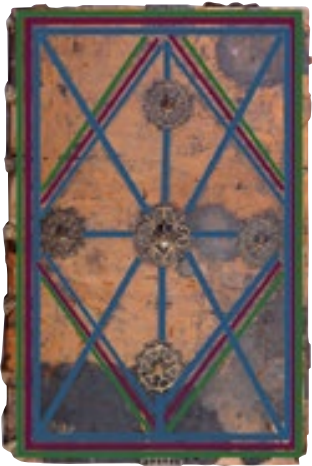

LC-1719-MNV1

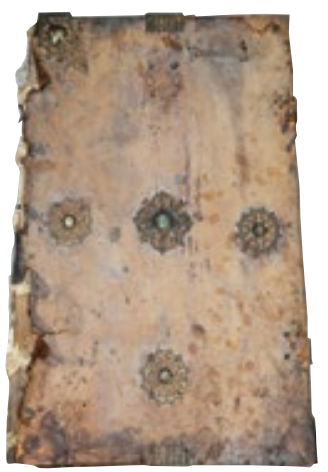

LC-1715-MNH

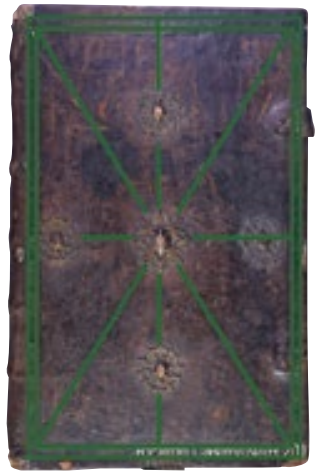

LC-1719-MNV2

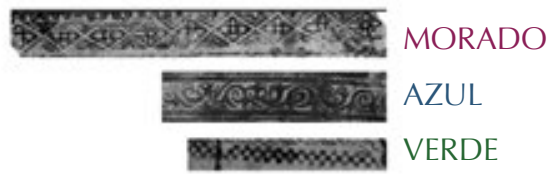

FIGURA 10. Decoración de gofrado con patrón compositivo realizado con tres hierros diferentes (Esquema: Tania Estrada 2013; fuente: Estrada, De la Garza y Velasco Castelán 2013b) que el LC-1714-MNV y el LC-1719-MNV2 también se encuentran decorados en el lomo con gofrado. Aunque no es claro por qué unos se decoraban con éste y otros no, se puede pensar que estas decisiones se tomaban de acuerdo con un criterio estético del encuadernador o bien a petición y gusto del comitente (Figura 10).

\section{Estado de conservación}

Podemos concluir que hubo cuidado en la selección de la calidad de los materiales y destreza en la manufactura del conjunto - texto manuscrito, letras iniciales y encuadernación - de los libros analizados, lo cual ha promovido, en términos generales, un buen estado de conservación tanto en su contenido como en la mayor parte de sus elementos estructurales, no así en su material de recubrimiento, especialmente en el lomo.

Del conjunto, el LC1714-MNV1 es el mejor conservado. Su principal deterioro es la pérdida de material de recubrimiento, en cabeza y pie del lomo, que dejó expuestos los endoses, nervios y cabezada. Las cabezadas tienen fractura en las bisagras anterior y posterior, por el debilitamiento de las fibras en dicha zona, así como abrasión mínima.

A su vez, el LC-1715-MNH parece ser el que se encuentra en peor estado de los cuatro: la piel, reseca y agrietada, presenta algunas manchas y faltantes; en la zona de las bisagras hay rotura, que derivó en la pérdida del lomo, por lo que quedan desprotegidos los elementos interiores, como la lomera, el endose y la estructura (Figura 11); los nervios han sufrido resequedad — de modo que algunos se encuentran quebradizos y rígidos- y rotura, tanto del hilo de la costura de los tres primeros cuadernillos, como en tres nervios en el área de la bisagra de la tapa anterior; hay faltante de un endose, en tanto que los demás se encuentran parcialmente despegados y, por último, hay rotura en la zona de la bisagra de la tapa anterior.

El LC1719-MNV1 presenta algunas reparaciones históricas, como zurcidos con hilo de fibras vegetales en el pergamino de los siguientes folios: $36 r$, $45 r$ y 71 r. En realidad, el principal deterioro se ubica en el material de recubrimiento, en el que, además de desprendimientos, muestra importantes faltantes y abrasión general, y, por el contacto con algún líquido que manchó y "quemó" la piel, un deterioro en la estructura de ésta. También hay pérdida parcial de la encuadernación: un faltante en la tapa posterior, correspondiente a la esquina superior derecha, así como desprendimiento de fragmentos en el ensamble de las esquinas de canto de pie en la tapa anterior. La pérdida de material de recubrimiento en el lomo promovió un desgaste y debilitamiento de las fibras en su costura y sus soportes, lo cual a su vez generó un desglose parcial: fractura y separación de algunos cuadernillos, así como faltantes, fractura y separación en el endose fraccionado. 


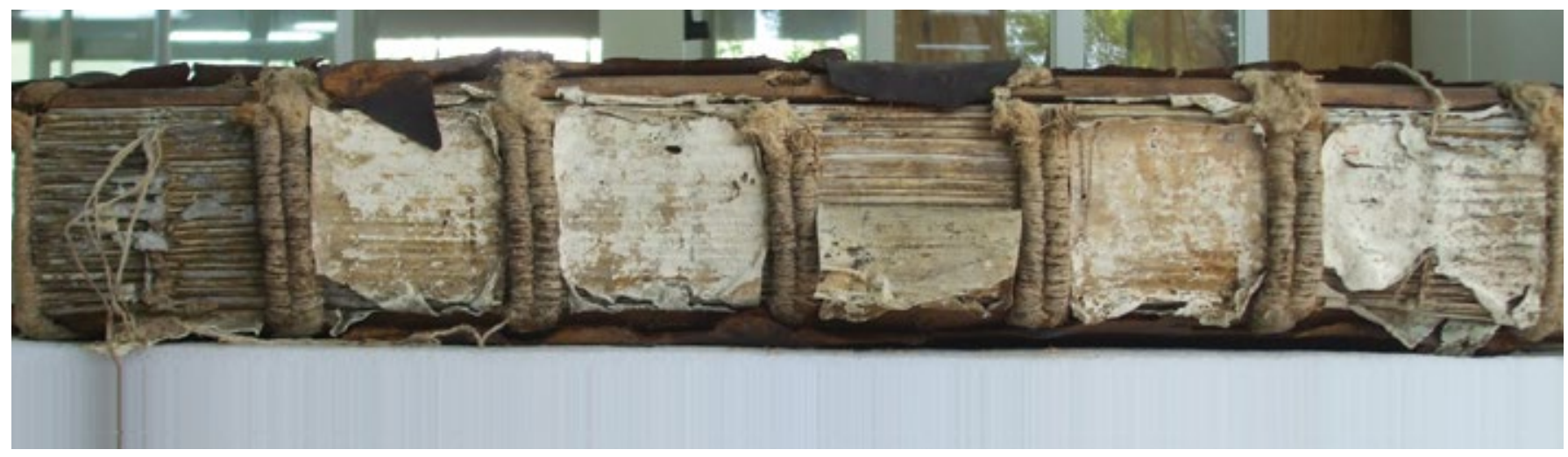

FIGURA 11. Elementos interiores expuestos por pérdida de lomo (Fotografía: Tania Estrada 2013; fuente: Estrada y De la Garza 2013:53).

Finalmente, el principal deterioro del LC-1719-MNV2 es la separación parcial en algunos cuadernillos de la lomera, aparte de que presenta pequeños faltantes en la piel del lomo, concretamente en la zona de las cofias, lo cual expuso la estructura y, a su vez, provocó un faltante parcial de las cabezadas, así como desprendimiento y fractura total del alma de las cabezadas. Una intervención, que inferimos se realizó hace algunos años, ocasionó un problema estético en la encuadernación: probablemente con la intención de proteger y "restaurar" la piel, se aplicó un líquido que la oscureció y que modifica su apariencia y textura, además de que dificulta apreciar el motivo gofrado original.

En general, observamos deterioros mínimos, más bien derivados tanto del formato y peso de los libros como del uso al que estaban sometidos. Debe subrayarse que los elementos estructurales y la estructura propiamente dicha que permiten el movimiento del libro y conservan su contenido son estables.

\section{Reflexiones finales}

A raíz del trabajo de investigación del LC-1715-MNV, del hallazgo del oficio de Antonio Cortés (Cortés 1915) y del desarrollo del proyecto "Estabilización de la colección de los libros de coro del Museo Nacional del Virreinato, Tepotzotlán" (Estrada, De la Garza y Velasco Castelán 2013b) se localizaron los quince libros de coro de fray Miguel de Aguilar y, además, se recopiló información sobre: a) los materiales constitutivos; b) la técnica de manufactura, y c) el estado de conservación del conjunto de volúmenes elaborados por el mismo copista entre los años 1700 y 1719: como primeros resultados del análisis comparativo de los primeros y la segunda, esbozados por el análisis de este cúmulo de datos, en el grupo se han identificado similitudes y diferencias de manufactura. A continuación se expondrán brevemente algunas reflexiones al respecto.

La presencia del colofón en los libros de coro es un rasgo de gran relevancia para el estudio de esta colección, no sólo porque se trata de un rasgo característico de los libros producidos por fray Miguel de Aguilar ${ }^{32}$, sino porque nos otorga datos sobre su manufactura: nombres de comitente, copista y fecha de elaboración. A su vez, el contar con un conjunto de libros producidos por un mismo artesano en un espacio y un tiempo específicos nos ha permitido analizarlos con el objeto de observar el desarrollo de la caligrafía del copista y de algunos elementos gráficos del conjunto.

Según hemos evidenciado de manera general en libros específicos como el LC-1701-MNV, De Aguilar participó como copista y como iluminador de letras iniciales (quebradas y lombardas), sin embargo, otros presentan motivos decorativos en sus letras capitulares mucho más elaborados (en algunos casos, habitadas), por lo que pareciera posible que se tratara de un trabajo de colaboración con uno o varios iluminadores.

En cuanto a la conformación del cuerpo del libro, en los cuatro volúmenes que conservan su primera encuadernación queda de manifiesto que existe un patrón de uso de cuadernillos formados por tres bifolios (seis hojas), aunque en algunos casos específicos hay cuadernillos de dos bifolios (LC-1715-MNH), cuya construcción siempre es la misma: talón doble adherido con adhesivo (aunque debe hacerse notar que ésta parece ser una característica que se observa en la mayor parte del acervo de libros de coro del MNV). La cantidad de cuadernillos y de fojas está determinado por el contenido de los libros. En tres de éstos, se presume, existían guardas lisas que, si bien ya no se conservan, originalmente formaban parte, podemos asegurar, del volumen, pues se tiene la evidencia del talón por medio del cual estaba pegada la otra foja (LC-1714-MNV, LC-1719-MNV1 y LC-1719-MNV2). El otro volumen conserva guardas de reúso.

Con base en el análisis comparativo superficial de los materiales y técnicas estructurales y decorativas de las encuadernaciones de los cuatro libros de coro que presentan

\footnotetext{
32 La mayor parte de los libros de coro de la Catedral de México y del
} MNV carece de colofón. 
la primera encuadernación, podemos argüir que su manufactura en este conjunto probablemente fue realizada por una misma persona o en un mismo taller: la estructura de los ejemplares es prácticamente la misma y todas cuentan con los mismos elementos estructurales y con materiales similares; los herrajes también tienen semejanzas y presentan la misma disposición; adicionalmente, identificamos tres motivos de carretillas utilizadas para gofrar los planos de piel, que se ocuparon indistintamente en tres de los volúmenes, con esquemas compositivos de motivos gofrados con algunas diferencias.

A pesar de estos resultados, será necesario, a la luz de la factura, la historia y el contenido del resto de la colección, analizar el conjunto de datos con mayor detalle. Por ejemplo, queda pendiente vincular el tema del contenido de los libros de coro con el registro de manufactura y el estado de conservación, análisis que seguramente será de gran riqueza para comprender los usos que tenían dentro de la comunidad religiosa. Un claro ejemplo de dicha correlación lo encontramos en el libro LC-1713-MNV: un sencillo registro de éste nos permitió observar que se encuentra reencuadernado y que presenta manchas de grasa en las esquinas inferiores de las hojas del cuerpo del libro, reparaciones a manera de costura del pergamino y manchas de cera y abrasión en las tintas. ¿Por qué la reencuadernación y el deterioro caracterizan este volumen?: el libro contiene un compendio de himnos que debían cantarse a lo largo de todo el año, por lo que es muy posible que este deterioro se deba al uso cotidiano. Conclusiones como ésta sólo pueden realizarse gracias a un trabajo interdisciplinario entre restauradores, musicólogos e historiadores, como el que, afortunadamente, se llevó a cabo durante el desarrollo del proyecto que aquí hemos presentado.

Se espera profundizar en el análisis de los cuatro libros para después comparar los otros libros que, de menor o mayor manera, presentan modificaciones en su estructura y primeras encuadernaciones. El objetivo general de este trabajo ha sido, como hemos venido exponiendo, determinar las características de la manufactura de los libros de coro de un fraile del convento de san Agustín que laboró entre 1701 y 1719 en la ciudad de México. Este modelo, o modelos, podrá ser un referente para el análisis de otros conjuntos de libros de coro. ${ }^{33}$

\section{Agradecimientos}

Se agradece al equipo de la Coordinación Nacional de Conservación del Patrimonio Cultural del Instituto Nacional de Antropología e Historia (CNCPC-INAH, México) que colaboró en el proyecto "Estabilización de la colección

\footnotetext{
${ }^{33}$ El informe presentado deriva de la ponencia "La conservación de los libros de coro copiados por fray Miguel de Aguilar a través del análisis de su manufactura y estudio de su historia de tránsito" (Estrada, de la Garza y Velasco Castelán 2013b).
}

de los libros de coro del Museo Nacional del Virreinato, Tepotzotlán": Diana Velázquez, Gisela Villanueva, Olivia Ibarra, Mayté Ruiz, Sandra Ordaz, Jeniffer Ponce, Dalila Terrazas, Carina Cruz, Marie Vander Meeren, Ricardo Castro y Víctor Gutiérrez.

Agradecemos a la doctora Martha Romero que nos haya permitido leer su artículo "Análisis e interpretación de los materiales empleados en la encuadernación de los libros impresos mexicanos del siglo XVI", el cual se publicará este año.

\section{Referencias}

\section{AHMNA-INAH}

1925 "Comunicación de traslado de 37 libros de coro", documento mecanoescrito [oficio], México, Fondo Museo Nacional de México (AHMNA-INAH), volumen 49, 13 de agosto de 1925, f. 124.

Bueno, Javier

2005 "La encuadernación de los libros de coro: las cubiertas de los cantorales de la abadía del Sacromonte de Granada", Boletín del Instituto Andaluz del Patrimonio Histórico, 13: 58-69.

CNCPC-INAH

1973 "Oficio de entrada del LC-1715-MNH al Departamento de Restauración del Patrimonio Cultural (actual CNCPC)", documento mecanoescrito [oficio], México, Archivo Histórico de la Coordinación Nacional de Conservación del Patrimonio Cultural (CNCPC-INAH), expediente (G/09-016-000/ ROT/2), 15 de octubre.

Cortés, Antonio

1915 "Lista de objetos que se pueden exhibir en el Museo Nacional", documento mecanoescrito, México, Archivo Fondo Biblioteca Nacional de México, caja 70, expediente 1597. Estrada, Tania y Patricia de la Garza

2013 "Propuesta de mínima intervención para el libro de coro de 1715 (LC-1715) a partir del estudio de los elementos estructurales y de las encuadernaciones de los libros de coro copiados por fray Miguel de Aguilar en el siglo XVIII", tesis de licenciatura en restauración, México, ENCRyM-INAH.

Estrada, Tania, Patricia de la Garza y Thalía E. Velasco Castelán 2013a "La conservación de los libros de coro copiados por fray Miguel de Aguilar a través del análisis de su manufactura y estudio de su historia de tránsito", ponencia presentada en el Primer Congreso de Conservación del Patrimonio Documental, 2-6 de septiembre, México, ENCRyM-INAH/CNCPCINAH/Adabi.

2013b "Proyecto de estabilización y digitalización de la colección de libros de coro del Museo Nacional del Virreinato", ponencia presentada en el Segundo Coloquio Internacional: Hacia la Preservación del Patrimonio Documental. Líneas de Trabajo en Materia de Conservación y Restauración en Bibliotecas y Archivos, 7-11 de octubre, UNAM-IIB/BN/HN/ ENCRYM-INAH.

Ferguson, George

1956 Signos y símbolos en el arte cristiano, Buenos Aires, Emecé. 
García Isac, Marta, Liudmilla Lidón y Josefa Sánchez 2010 "Restauración del Cantoral del Aula Salinas de la Universidad de Salamanca", Encuadernación de Arte, 38 (3): 37 50 .

Hellinga, Lotte

2000 "Fragments Found in Bindings and their Role as Bibliographical Evidence", en David Pearson (ed.), For the Love of Binding: Studies in Bookbinding History, Londres/New Castle/Delaware, The British Library/Oak Knoll Press, 13-33.

Lara Cárdenas, Juan Manuel 1996 "Los libros de coro del Museo Nacional del Virreinato", Tepotzotlán ayer y hoy: Trigésimo Aniversario, Museo Nacional del Virreinato-INAH, México, INAH: 50-56.

Ligatus

2011 Ligatus, A research centre of the University of the Arts London with projects in historical libraries and archives, documento electrónico [página web] disponible en [www.ligatus.org.uk], consultado el 29 de agosto de 2013.

Marín, Javier

2007 "Música y músicos entre dos mundos: la Catedral de México y sus libros de polifonía (siglos XVI-XVIII)", tesis de doctorado en historia y ciencias de la música, Universidad de Granada.

Martínez de Sousa, José 2004 Diccionario de bibliología y ciencias afines, Gijón, Trea. Olivé Negrete, Julio César y Bolfy Cottom

2003 INAH, Una historia. Leyes, reglamentos, circulares y acuerdos, vol. 1, México, INAH.

Pedraza Gracia, José Manuel, Yolanda Clemente San Román y Fermín de los Reyes Gómez

2003 El libro antiguo, Madrid, Síntesis.

Pérez, Mónika

2009 "Libros de coro de canto Ilano, manuscritos de la Catedral de México, análisis y propuesta metodológica para su dictamen", tesis de licenciatura en restauración, México, ENCRYM-INAH.

RAE

2013 "Copista", en Diccionario de la lengua española, RAE (Real Academia Española), documento electrónico [página web] disponible en [http://www.rae.es], consultado en abril del 2013.

Romero, Martha Elena

En prensa "Análisis e interpretación de los materiales empleados en la encuadernación de los libros impresos mexicanos del siglo XVI", en Marina Garone (coord.), De la materialidad de la conservación del patrimonio bibliográfico y archivístico, México, UNAM.
Romero de Terreros, Manuel

1943a Encuadernaciones artísticas mexicanas, siglos XVI al XIX, México, Dirección de Acción Social.

1943b La iglesia y convento de san Agustín, México, IIEsUNAM.

Rubial García, Antonio

1989 El convento agustino y la sociedad novohispana (15331630), México, UNAM.

2005 "Los conventos mendicantes", en A. Rubial García (coord.), Historia de la vida cotidiana en México, vol. II, México, El Colegio de México/FCE, 169-192.

Ruiz García, Elisa 2002 Introducción a la codicología, Madrid, Fundación Germán Sánchez Ruipérez.

Ruiz Zavala, Alipio

1984 Historia de la provincia agustiniana del santísimo nombre de Jesús, México, Porrúa.

Ruiz Castañeda, María del Carmen, Luis Mario Schneider y Miguel Ángel Castro Medina (comps. y eds.)

2004 La Biblioteca Nacional de México: testimonios y documentos para su historia, México, IIB-UNAM.

Salgado Ruelas, Silvia 2010 Libros de coro conservados en la Biblioteca Nacional de México, México, Adabi.

Tello, Aurelio 1988 "Plegarias al oído", en Miguel Hernández (coord.), Tepotzotlán. La vida y la obra en la Nueva España, México, Sociedad de Amigos de Museo Nacional del Virreinato/Bancomer, 248-256.

Velasco Castelán, Thalía E.

2005 "Diagnóstico de la colección de libros de coro del Museo Nacional del Virreinato (MNV)", documento mecanoescrito, Tepotzotlán, CNCPC-INAH.

2009a "Los libros de coro de la Catedral Metropolitana: una propuesta para interpretar su uso a través de sus modificaciones en la conservación-restauración en el INAH", en Renata Schneider (coord.), La conservación-restauración en el INAH. El debate teórico, México, INAH, 97-115.

2009b "Estudio material de un libro de coro: un acercamiento a su manufactura", en Luis Fernando Guerrero Baca (coord.), Conservación de bienes culturales: acciones y reflexiones, México, INAH, 347-364.

2012 "La construcción y el valor del patrimonio documental en el marco legislativo e institucional mexicano (19142012)", tesis de maestría en historia aplicada, Costa Rica, Sistema de Estudios de Posgrado-UNA. 


\section{Resumen}

Las referencias documentales y bibliográficas en torno de la manufactura de los libros de coro en la Nueva España son escasas, limitación que, aunada a la localización de un conjunto de libros de coro copiados por un mismo copista novohispano, fray Miguel de Aguilar, despertó el interés por realizar el registro y el estudio de estas obras, temáticas centrales del presente INFORME.

Como parte de ello, y dado que los ejemplares de este conjunto se conservan en distintos acervos del Instituto Nacional de Antropología e Historia (INAH): los museos nacionales del Virreinato (MNV) y de Historia (MNH), y la Biblioteca Nacional de Antropología e Historia (BNAH), todos en México, se decidió llevar a cabo un análisis comparativo de sus materiales constitutivos y sus características constructivas, cuyas similitudes y diferencias arrojan valiosa información sobre la tradición de encuadernación mexicana en el siglo XVIII.

\section{Palabras clave}

libros de coro; manufactura; encuadernación; siglo XVIII; México

\section{Abstract}

Documental and bibliographic references on the production of choir books from New Spain are scarce. This restriction, coupled with the finding of a collection of books produced by the Novo Hispanic copyist Miguel de Aguilar, aroused the authors' interest to carry out the registration and study of these works, central themes of this REPORT.

As part of the research project it was decided to carry out a comparative analysis, also because the respective items of the collection are stored in different archives of the Instituto Nacional de Antropología (National Institute of Anthropology and History, INAH): el Museo Nacional del Virreinato (National Museums of Mexican Viceroyalty, MNV) el Museo Nacional de Historia (National Museum of History, MNH), and the Biblioteca Nacional de Antropología e Historia (National Library of Anthropology and History; BNAH), all in Mexico. By comparing the constituting materials of the different copies as well as their constructional properties, both similarities and differences came to the light, which yield valuable information about the tradition of Mexican bookbinding in the eighteenth century.

\section{Key words}

choir books; manufacture; book-binding; eighteenth century; Mexico

Título en Inglés: A First Approach towards the Binding of Choir Books copied

by Friar Miguel de Aguilar in New Spain

Postulado/Submitted 13.01.2014

Aceptado/Accepted 04.08.2014 